\title{
AC 2011-439: DESIGN OF A WIRELESS SENSOR AND ACTUATOR NET- WORK FOR ENERGY MANAGEMENT AT HOME
}

\author{
Chao Chen, Indiana University - Purdue University Fort Wayne
}

Dr. Chao Chen is a Computer Engineering Assistant Professor with the Department of Engineering at Indiana University - Purdue University Fort Wayne. She received her M.S. and Ph.D. degrees from Georgia Institute of Technology in 2003 and 2005, respectively. She also holds B.E. and M.E. degrees from Shanghai Jiao Tong University, Shanghai, China in 1998 and 2001, respectively. Her current research interests include wireless opportunistic networks, wireless ad hoc and sensor networks, cognitive radio networks, network security, modeling and performance evaluation of communication networks.

\section{Carlos Pomalaza-Raez, Indiana University Purdue University, Fort Wayne}

Carlos Pomalaza-Rez is an Electrical Engineering Professor at Indiana-Purdue University, Indiana, USA, and a Visiting Professor at the University of Oulu, Finland. He received his BSME and BSEE from Universidad Nacional de Ingeniera, Lima, Peru in 1974, and his MS and PhD in Electrical Engineering from Purdue University, West Lafayette, Indiana, in 1977 and 1980, respectively. He has been a Faculty Member of the University of Limerick, Ireland, and of Clarkson University, Potsdam, New York. He has also been a member of the technical staff at the Jet Propulsion Laboratory of the California Institute of Technology.

Edwin Chobot, Purdue University, Fort Wayne

Daniel B Newby, Indiana University, Purdue University, Fort Wayne

Renee Kathleen Chandler, Purdue University, Fort Wayne

Renee is a senior Computer Engineering student at Purdue University in Fort Wayne, Indiana. She is excited to graduate in May 2011 and pursue a career in firmware development and embedded systems.

Ms. Nusaybah Abu-Mulaweh

(C)American Society for Engineering Education, 2011 


\section{Design of a Wireless Sensor and Actuator Network for Energy Management at Home}

\section{Introduction}

In a world of rising energy costs and dwindling natural resources capable of producing energy, people and businesses are starting to look for better ways to help reduce their increasing electric bills. One way of reducing these costs is to monitor, in real time, how much power is being consumed and from these data make informed decisions about how to manage the electrical devices being powered. A system that can give users an estimate of how much energy is being, has been, and might be consumed will allow them to adjust their habits and lower the costs.

In this paper, we describe a capstone senior design project that designs, builds, and tests a wireless sensor and actuator network for monitoring the energy use of alternating current (AC) appliances in a home environment. The measured energy use of individual appliances can be displayed through a user visual interface in real time; so that users can easily understand their electricity usage patterns and adapt their behavior to reduce their energy consumption and costs. Moreover, users are able to remotely control the power on/off of individual devices to actively control the power use of certain appliances. The system allows for inexpensive monitoring of home energy use and illustrates a practical way to control the energy consumption through user interaction.

The remainder of this paper is organized as follows. The overview of the project and its differences with some products in the market are described in Section 2. The detailed design of the system is explained in Section 3. Section 4 analyzes some important aspects of the system design. Section 5 concludes this paper.

\section{Project Description}

In the capstone senior design course in the Department of Engineering at Indiana University Purdue University Fort Wayne (IPFW), students complete a major design project by taking a required two-semester course sequence. All participating students are teamed in groups and work on different design projects. Each team is supervised by a faculty member. In the first semester, students go through the processes of problem statement, basic conceptual design and evaluation, and detailed final design. The prototype development, test, and analysis are done in the second semester. At the end of both semesters, the senior design group is required to summarize their progress and results in the form of final technical reports and give a formal presentation to the faculty, students, industrial sponsors, and the general public. Through the design and evaluation process, students gain experience in the process of practical electrical and computer system design from an initial concept through the final design.

\section{Project Overview}

This specific capstone senior design project started in Fall 2010, and the team has two Computer Engineering students and two Electrical Engineering students. The goal of this project is to design, build, and test a wireless sensor and actuator network for monitoring the energy use of 
$\mathrm{AC}$ appliances in a home environment. The wireless sensor and actuator network consists of multiple measurement nodes and a central server module, where the measurement nodes have two-way communication with the central server module. Each measurement node in the network is connected to and reads the energy use of one AC appliance, and wirelessly reports the readings to the central server module for processing. The server displays the readings from these nodes through a user visual interface in real time. This system can help users better understand their electricity usage patterns and adapt their behavior to reduce their energy consumption and costs.

Figure 1 shows an application of this wireless energy monitoring system in a home scenario, where the measurement nodes are connected to major home appliances in different rooms, and the central server module displays the energy consumption of these appliances on a computer screen. If the server node or the computer is accessible through the Internet or the cellular network, users can also remotely track their home energy usage online.

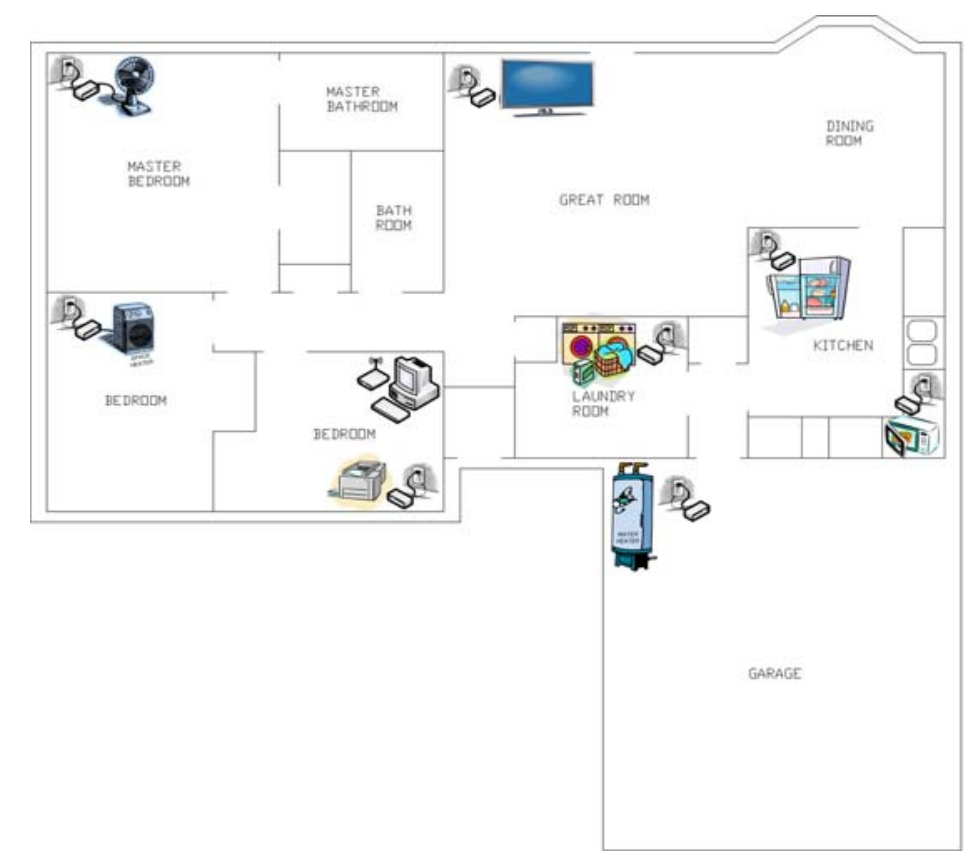

Figure 1. A wireless energy monitoring system at home.

To make the system design and testing feasible for a senior design project, our group decided to design and implement a system prototype with two measurement nodes and one central server module, where the nodes communicate directly to the server and the server displays the measurement results through the computer. The system prototype will be deployed and tested indoor in a typical home environment in the United States. The communication distance from the measurement nodes to the central server module is within 15 meters.

\section{Remote Power On/Off Control}

Another main objective of this project is to allow users to actively control the energy usage of certain devices through the wireless sensor and actuator network. To illustrate a simple method without getting into the functionalities of specific appliances, we choose power on/off control in the following application scenario. Most electronic products, even if turned off, will continue to 
draw power from a standard electrical outlet unless the device is manually unplugged. This power consumption is called "standby power." Although individual electronic products might not draw much power to be noticed while in standby mode, the average American family has almost forty devices constantly consuming power. The standby power consumption of these devices accounts for almost $10 \%$ of the whole household electricity use ${ }^{1}$. Because of this, our design will integrate an actuator into each measurement node that automatically turns on and off the power supply to the products remotely. The control method can be time-based or measurement-based. In the time-based control, a user sets certain time frames that he/she wants to cut off the power of some devices. This would eliminate the standby power consumption in those nodes during those time frames. In the measurement-based control, the system will determine the standby status of certain devices and automatically cut off the power if a device has stayed in the standby mode for a long time.

The remote on/off control can also be used in other manners to further reduce energy. For example, the air conditioner and fans can be turned on and off remotely based on the inputs from temperature sensor readings; lights can be turned on and off remotely based on the inputs from light sensor and motion sensor readings. The design of these appropriate control mechanisms, however, depends on specific appliances and the habits of individual users. Therefore, in our project, we specifically target the standby power to illustrate the feasibility and functionality of the on/off control.

\section{Related Products}

Currently there are several products already on the market that can be used for power monitoring purpose. For example, the Pacific Gas and Electric Company (PG\&E) in California has started a program to replace conventional meters by the SmartMeter ${ }^{2}$. The SmartMeter records the whole house electricity consumption on an hourly basis. The main goal of the SmartMeter technology is to enables PG\&E to set pricing that varies by season and time of the day, rewarding customers who shift energy use to off-peak periods. TED (The Energy Detective) ${ }^{3}$ measures home energy usage through residential electric panels and updates real-time monitoring results every second. TED displays the results on an LCD screen and/or a local or remote computer using its datalogging software or Google PowerMeter ${ }^{4}$, a free energy monitoring software. Kill-A-Watt ${ }^{5}$ is a device which monitors the amount of electric consumption of a connected appliance by the kilowatt-hour and displays it on an LCD display. Users can calculate the electrical expenses by the day, week, month, or year.

Compared with the above products on the market, our proposed energy monitoring system aims to monitor the energy consumption of individual appliances (like Kill-A-Watt) and provides realtime measurement results (like TED), so that users know where they can save more energy and are able to get immediate feedback on their power usage. Moreover, our system is capable of controlling individual appliances automatically or remotely through a computer. Although simple, this on/off control enhances the wireless network from monitoring only to including the actuator part, which extends the capability of the whole system and makes our design different from other products on the market. If the power on/off control is feasible, there should be no problem adding other more complicated control mechanisms. Due to the limitations in time and resources, our senior design project will only focus on a power on/off control mechanism. 


\section{Project Design}

Our system prototype consists of two measurement nodes and one central server module, where the nodes measure the electricity consumption of the connected appliances and directly report the results to the server through wireless communication; and the central server module displays the readings from these nodes through a user visual interface via a computer.

\section{Design Overview}

The group first generated ideas for the design and decided that the measurement nodes and the central server module should have the following major components.

Measurement Node Components:

- An AC-to-DC power supply that will supply power to the energy monitoring circuitry.

- A current to voltage conversion circuit that will facilitate the measurement of the current flowing to the AC device.

- An energy measurement circuit that samples the continuous current and voltage signals in order to compute the energy consumption of the attached device.

- A microcontroller that performs the necessary calculations and then send the resulting data to a transceiver for transmission.

- A wireless transceiver that handles transmissions between the node and the server.

- An actuator that allows the user or the server to control the power on/off of the attached AC device.

- Indicators that alert the users of the operating status of a measurement node.

Central Server Module Components:

- A wireless transceiver for wireless communication with the measurement nodes.

- A USB port that can be used to connect to a personal computer.

- A graphical user interface (GUI) that allows the user to view the energy usage in realtime and track past power usage.

\section{Detailed Component Design}

In the following paragraphs, we give the detailed design of the major components in the measurement nodes and the central server module.

\section{$\underline{\text { AC-to-DC Power Supply }}$}

In our design, each measurement node will be plugged into a standard NEMA 5-15 electrical outlet. The AC device will then be plugged into the measurement node. The circuitry used to measure the power will need to be powered by DC voltage. This may include several different $\mathrm{DC}$ voltage levels, such as $3.3 \mathrm{~V}$ and $5.0 \mathrm{~V}$. The plan for our design is to tap the AC power and convert it the DC power required by the measurement node's internal circuitry. 
Our AC-to-DC power supply consists of three stages. The first stage is a step-down transformer that scales down the AC voltage by a specified factor, such as 10:1. This has the benefit of allowing access to a smaller AC voltage that can be used for our voltage measurements. Additionally, the voltage output by the secondary coil of the transformer is electrically isolated from the primary coil (main AC power supply). Next, the secondary AC signals are rectified by using a full-wave bridge rectifier. In the last stage, the voltage waveform is converted to DC voltage using a filter and voltage regulators. Figure 2 below shows the schematic of the AC-toDC power supply that has been adopted in our design.

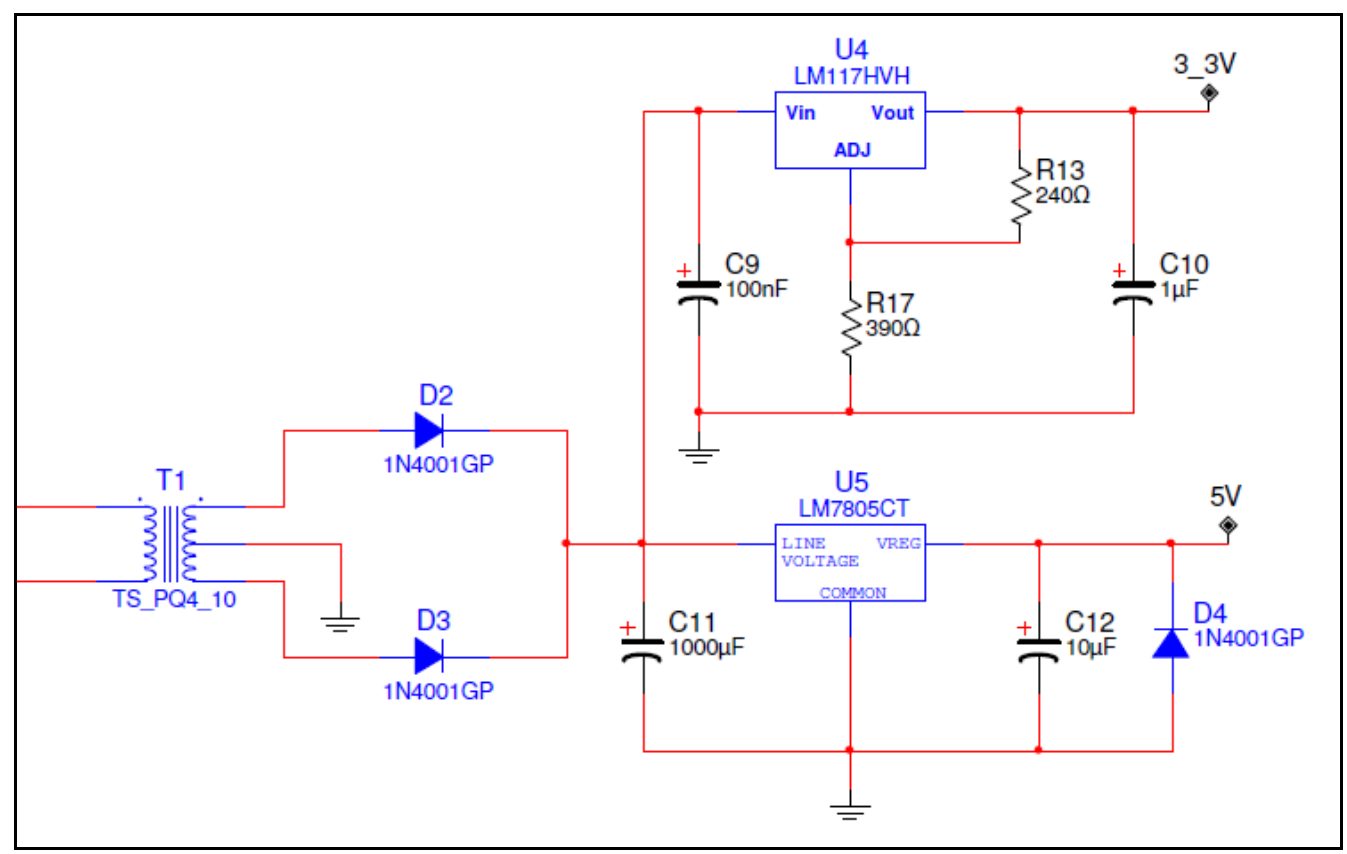

Figure 2. The schematic for step-down transformer power supply.

\section{Current-to-Voltage Conversion}

To calculate the real-time energy consumption of a connected AC device, we need to measure the input voltage and the current flowing through the connected device. Since analog to digital converters require an input voltage for their conversions, not a current, the current passing through the load appliance must be converted to a voltage before recordings can occur. After the recordings are taken, the voltage measurement will be converted to a current measurement and power calculations can take place. We considered the following two options for the current-tovoltage conversion:

- Hall Effect Sensor: A Hall Effect sensor is a transducer whose output voltage varies with changes in magnetic field. Changes in magnetic field occur if the current flowing through the load appliance changes. By using a Hall Effect sensor, the interference with the actual load can be kept to a minimum.

- Low Impedance Resistor: A low impedance resistor can be used in series with the load to determine the current flowing through the load by using Ohm's law. While this is simple, it slightly alters the voltage delivered to the load because the resistor reduces the voltage delivered to the load. 
We choose to use the low impedance resistor in our design since it takes up less space and is less expensive than the Hall Effect sensor. Specifically, we select the Panasonic ERJM1WS $15 \mathrm{~m} \Omega$ resistor ${ }^{6}$, which costs $\$ 1.18$ each.

\section{Energy Measurement}

The power supplied by a NEMA 5-15 standard electrical outlet is delivered as a sinusoidal waveform with a frequency of $60 \mathrm{~Hz}$. According to the Nyquist-Shannon sampling theorem, a sampling rate greater than $120 \mathrm{~Hz}$ is required to accurately measure the voltage and current through the connected appliance. During each sample period, the analog samples will be converted into a digital signal with an analog-to-digital converter (ADC). Additionally, the voltage and current will need to be sampled simultaneously in order to accurately calculate the instantaneous power usage. This requires that our design includes two separate ADCs.

Another consideration in the implementation of the digital conversion is the resolution of the conversion. The resolution of the converters will determine the precision of the measurement. There are many ADC chips available with resolutions between 8 to 16 bits. The power supplied by a NEMA 5-15 standard electrical outlet has rated maximum voltage of $125 \mathrm{~V}_{\text {rms }}$ and maximum current of 15A. Table I below shows the relationship between the number of bits and the corresponding precision of the measurements.

Table I. The number of bits of an ADC versus the resulting resolution of the power measurement

\begin{tabular}{|c|c|c|c|c|}
\hline $\begin{array}{c}\text { Number } \\
\text { of Bits }(\mathrm{N})\end{array}$ & $\begin{array}{c}\text { Number of } \\
\text { Discrete } \\
\text { Values }\left(2^{N}\right)\end{array}$ & $\begin{array}{c}\text { Voltage Resolution } \\
\text { for } \\
V_{p p}= \pm 125 \sqrt{2} \approx 353\end{array}$ & $\begin{array}{c}\text { Current Resolution } \\
\text { for } \\
I_{p p}= \pm 15 \sqrt{2} \approx 42\end{array}$ & $\begin{array}{c}\text { Power } \\
\text { Resolution } \\
\mathrm{P}=\mathrm{VI}\end{array}$ \\
\hline 8 & 256 & $1.38 \mathrm{~V}$ & $164 \mathrm{~mA}$ & $226 \mathrm{~mW}$ \\
\hline 10 & 1024 & $340 \mathrm{mV}$ & $41 \mathrm{~mA}$ & $14 \mathrm{~mW}$ \\
\hline 12 & 4096 & $86 \mathrm{mV}$ & $10 \mathrm{~mA}$ & $0.86 \mathrm{~mW}$ \\
\hline 16 & 65536 & $5.4 \mathrm{mV}$ & $0.65 \mathrm{~mA}$ & $3.5 \mu \mathrm{W}$ \\
\hline
\end{tabular}

We choose the Analog Devices AD71056 energy metering chip ${ }^{7}$ to measure the power consumption. This chip contains two 16-bit ADCs for the voltage and current signal inputs, which provides very precise power measurements. The analog input range is fully differential ( $\pm 30 \mathrm{mV}$ for the current signal and $\pm 165 \mathrm{mV}$ for the voltage signal), which does not require adding the DC offset to the AC signals that are necessary for single-ended analog input ranges such as $0-5 \mathrm{~V}$. A high sampling rate of $450 \mathrm{kHz}$ is used, which is well above the minimum sampling rate required. There are three output signals specifying the power measurement: One high frequency output supplies instantaneous real power, and two low frequency outputs supply average real power. This chip requires a small amount of power $(20 \mathrm{~mW})$ and is priced at $\$ 2.63$ each.

The complete energy measurement circuit is shown in Figure 3, where the purpose of the voltage signal conditioning is to scale down the AC voltage signal to the specified input range of $\pm 165 \mathrm{mV}$. The AC current signal will be generated using a low-impedance sense resistor (R11 in Figure 3) and the current signal conditioning limits the range of the input range for this signal to 
$\pm 30 \mathrm{mV}$. Both signals are band limited with a low-pass filter with $3 \mathrm{~dB}$ cut-off frequency at $5.3 \mathrm{kHz}$. This will reduce any aliasing of the signals when sampled by the ADCs. On the other hand, the attenuation to the $60 \mathrm{~Hz}$ input signals will be negligible.

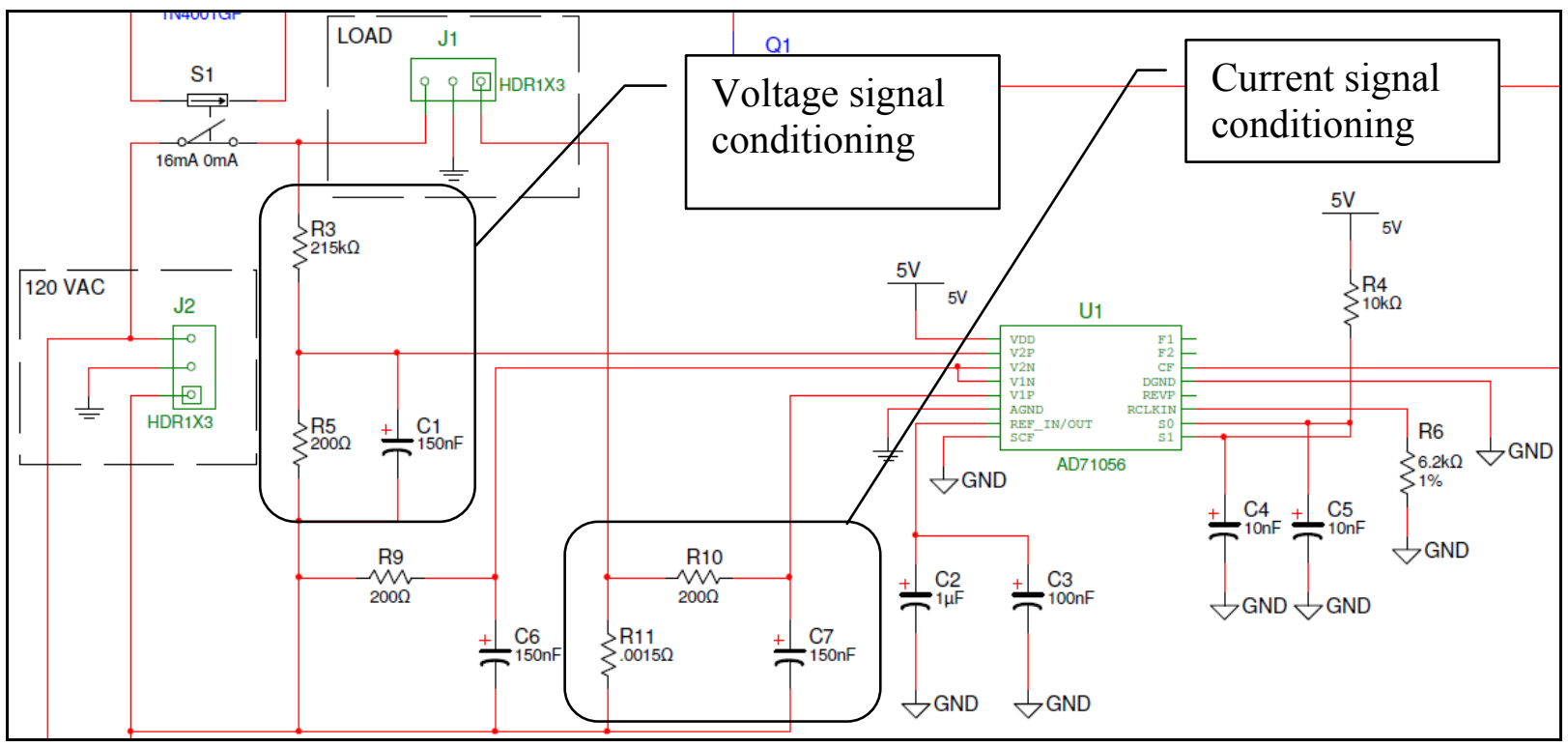

Figure 3. The schematic for the energy measurement circuit.

\section{$\underline{\text { Wireless Transceiver }}$}

Our system is targeted for indoor use at a typical American home; therefore a short-distance wireless communication system is acceptable. Two types of wireless communication standards are suitable for this application: IEEE 802.15.1 (Bluetooth) ${ }^{8}$ for medium rate wireless personal area networks (WPAN) and IEEE 802.15.4 (ZigBee) ${ }^{9}$ for low rate WPANs, both operating in the 2.4GHz unlicensed industrial, scientific, and medical (ISM) frequency band.

IEEE 802.15.1 is adapted from Bluetooth, which specifies short-range RF-based connectivity for portable devices. Bluetooth is designed for small and low cost devices with low power consumption. Since Bluetooth is geared towards handling voice, images, and file transfer, it has a data transfer rate on the order of $1 \mathrm{Mbps}$ with a relatively complex protocol. The operational range for Bluetooth is around 10 meters. With amplifier antennas its range can be boosted to 100 meters, but with higher power consumption.

IEEE 802.15.4 handles low-cost, low-speed ubiquitous communication between devices. It is designed for equipments that need a battery life as long as several months to several years but do not require a data transfer rate as high as those enabled by Bluetooth. The 802.15.4 compliant devices have a transmission range between 10 and 75 meters and a data transfer rate of $250 \mathrm{kbps}$ (if operating at $2.4 \mathrm{GHz}$ frequency band). 802.15.4 supports a basic master-slave configuration suited to static star networks of many infrequently used devices that talk via small data packets. Compared with Bluetooth, 802.15.4 is more power-efficient because of its small packet size, reduced transceiver duty cycle, reduced complexity, and strict power management mechanisms such as power-down and sleep mode. 
For our design, we have chosen the IEEE 802.15.4 standard, and specifically, the XBee 802.15.4 OEM RF module ${ }^{10}$ from DigiKey as the wireless transceiver. It can connect directly to a microcontroller with a UART interface. With a chip antenna, it operates up to 30 meters indoor. The transmission range can be further increased to 90 meters by using a whip antenna. The XBee module has a low maximum transmit power of $1 \mathrm{~mW}$ and a high receiver sensitivity of $-92 \mathrm{dBm}$. Each transceiver can be uniquely identified by its media access control (MAC) address. The $\mathrm{XBee}$ transceiver is priced at $\$ 19.00$ each.

\section{Actuator}

An actuator will be placed in each node with which the user can turn on or off the current flowing through the load. We choose a solid state relay (S1 in Figure 3) for this purpose. The specific part we picked is the Sharp Microelectronics S216S02F solid state relay ${ }^{11}$, which costs $\$ 6.45$ each.

\section{$\underline{\text { Indicators }}$}

The measurement nodes will have some visual indicators to alert the user of the status of the node. We choose the BIVAR SMP4 tri-color LED ${ }^{12}$ for the following indications:

- Green: The measurement node is functional.

- Yellow: The measurement node is transmitting data to the server.

- Red: The power to the load device has been shut off.

\section{Microcontroller}

The measurement nodes need a microcontroller to do simple processing on the sampled power measurement data from the energy metering chip, turn on or off the solid state relay, and control the flow of the data and control message. We choose Silicon Labs C8051F353 microcontroller ${ }^{13}$ with a built-in 16/24 bit ADC in our design.

\section{Graphical User Interface}

The graphical user interface (GUI) must display the energy usage measurement in real-time and provide for the control of the power on/off of the appliances through the measurement nodes. We have considered the following features that can be included in the GUI:

- Power usage display dial

- Pushbutton for on/off

- Histogram with hourly, daily, and weekly options

\section{System Design Summary}

Our system prototype has two measurement nodes and a central server module. Each measurement node will be plugged into a standard NEMA 15-5 electrical outlet. An AC device will then be plugged into the node for power measurement. Each measurement node contains the components necessary to measure the power consumption, wirelessly transmit the information to 
the central server module, and control the power on/off of the connected device. In order to measure the power consumption, a voltage divider network and low impedance current sense resistor are connected to an energy metering chip. The energy metering chip samples the voltage and current signals, calculates the power consumption, and outputs a pulsed digital signal with a frequency related to the power consumption. The microcontroller then samples the pulsed digital signal from the energy metering chip and converts to power. The microcontroller also integrates the power measurement samples to get the energy consumption and reports to the server through the wireless transceiver. The microcontroller also has the capability of controlling the power delivered to the connected device with a solid-state relay. An indicator LED will identify the node's current mode of operation, and a reset button will allow the user to restore power to a device that is currently off. The layout of the node is shown in Figure 4 below.

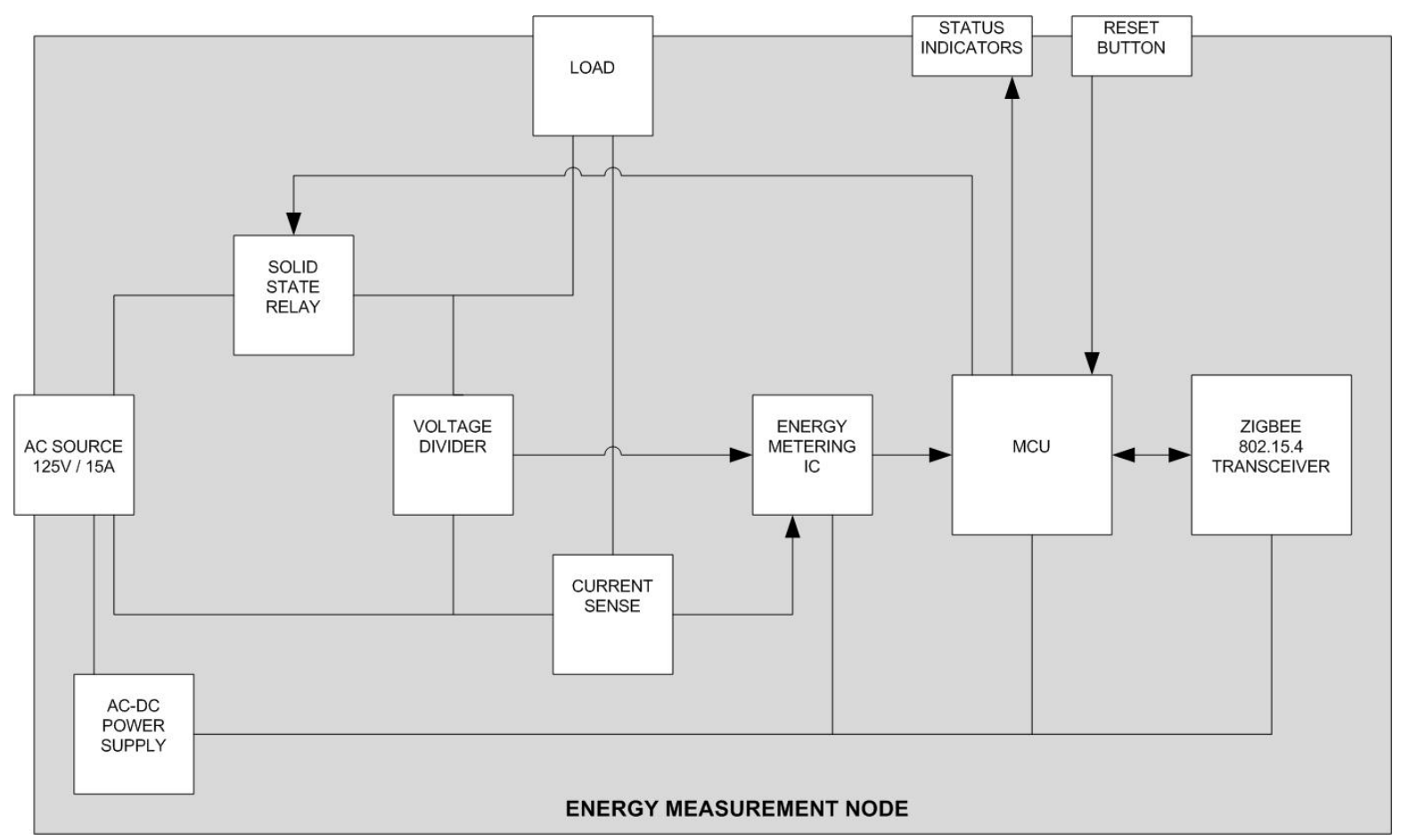

Figure 4. The block diagram of a measurement node.

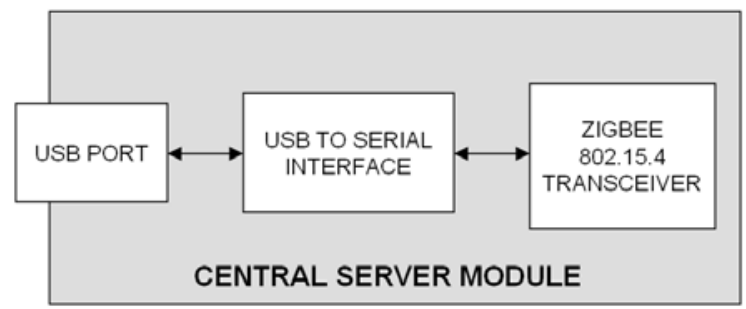

Figure 5. The block diagram of the central server module.

The central server module will receive the power measurements from each of the nodes and forward the measurements to the computer for display. The measurement data is received through a ZigBee transceiver and passed directly to the computer program through the USB port. The power on/off signals from the computer program are passed to the ZigBee transceiver 
through the USB port. The components in the central server module are powered through the USB port as well. Figure 5 shows a block diagram of the main components included in the central server module. We use the XBee Explorer Dongle unit ${ }^{14}$ from the SparkFun Electronics. This unit has a USB UART interface IC device (FT232RL) that interfaces between the XBee transceiver and the USB port. It also equipped with a USB port and a dock for the XBee transceiver. The XBee Explorer Dongle unit costs for \$24.95.

\section{System Analysis and Discussions}

In this section, we discuss several important aspects of this system prototype design, including real-time requirement, reliability, measurement node power consumption, and cost analysis.

\section{Real-Time Requirement}

To calculate the energy usage accurately from power measurement samples, we need to have the nodes sample the power measurement frequently, e.g., once per second or faster. If the power consumption of an appliance is almost constant across several sample intervals, the data sampling rate can be reduced. We plan to test the variability of the power consumption and set a reasonable sample rate accordingly.

Generally, to allow the users to view their energy use in real-time, it is sufficient to update the GUI data every minute. The data report rate from the measurement nodes can be set to the same rate with some retransmission mechanism under data losses. However, this data report and update rate can be adjusted according to the electrical device that a node is connected to. For example, the data report rate can be set to high when the microwave is on and low when the microwave is off. There are two advantages of this adaptive report rate control: First, the nodes can switch to a lower transmission frequency to reduce the power consumption. Second, there

will be fewer packets transmitted over the wireless medium, which leads to less collision and higher accuracy of the whole system.

\section{Reliability}

Since there are two measurement nodes in the network and both need to report the energy usage, the data reporting of the two nodes needs to be coordinated to avoid or reduce signal collision. To begin with, we plan to test the following options, and then evaluate which one would work best:

- The first option is to have the nodes transmit the results whenever they are ready. This may cause a collision. Therefore, some collision avoidance mechanism may be needed.

- The second option is to have the central server module poll the individual nodes for measurement results. This can avoid a collision but it adds the transmission overhead.

We can also explore the use of the contention free period (CFP) designed for real-time applications in the IEEE 802.15.4 medium access control protocol to evaluate the data latency in our project. 
Furthermore, other wireless signals in the house, if operating in the same frequency band, may interfere with the wireless signal transmissions of the system ${ }^{15,16}$. We plan to test the effect of these signal interference (e.g., WiFi signals) on the performance of the system.

\section{Node Power Consumption}

Table II shows the breakdown of the power consumption of the major components in the measurement node: the energy metering chip, the wireless transceiver, and the microcontroller. It can be seen that compared with other components, the wireless transceiver consumes much higher energy in transmit, receive, or idle mode. Therefore, the power consumption of the node does not vary much as the reporting rate changes. However, the power consumption of the measurement node can be reduced significantly in the sleep mode, which is pin-controllable. Therefore, we will explore the sleep mode in the testing phase to see how much power it can save for the wireless transceiver. Moreover, the energy consumption of the power metering chip can be further reduced with the on/off control. If the node is switched to be off, the power supply to the power metering chip can also be switched off, so that it does not consume any power in the off period. We will explore the on/off control of the power metering chip in our tests as well.

Table II. Power consumption of the major components in the measurement node.

\begin{tabular}{|l|c|r|}
\hline Node Component & Current and Voltage & Power Consumption \\
\hline Power metering chip & $4 \mathrm{~mA}$ @ 5 V & $20 \mathrm{~mW}$ \\
\hline Wireless transceiver & transmit mode: 45 mA @ 3.3 V & $148.5 \mathrm{~mW}$ \\
\cline { 2 - 3 } & idle/receive mode: 50 mA @ 3.3 V & $165 \mathrm{~mW}$ \\
\cline { 2 - 3 } & sleep mode: $<10 \mu \mathrm{A}$ or $<50 \mu \mathrm{A}$ & $<0.2 \mathrm{~mW}$ \\
\hline Microcontroller & $5.8 \mathrm{~mA} @ 3.3 \mathrm{~V}$ & $19.14 \mathrm{~mW}$ \\
\hline
\end{tabular}

\section{Cost Analysis}

Table III and Table IV list the costs of the major components of the measurement node and the central server module, respectively. The cost of the system prototype is estimated to be less than $\$ 250$, including an initial estimation of $\$ 43.95$ for the central server module and $\$ 94.50$ for each measurement node.

Table III. Cost of the major components in the measurement node.

\begin{tabular}{|l|r|}
\hline \multicolumn{1}{|c|}{ Component } & \multicolumn{1}{|c|}{ Price } \\
\hline AC-to-DC power supply circuit & $\$ 8.63$ \\
\hline energy measurement circuit (including the power metering chip) & $\$ 7.11$ \\
\hline XBee wireless transceiver & $\$ 19.00$ \\
\hline C8051F35x microcontroller & $\$ 9.77$ \\
\hline tri-color LED & $\$ 1.08$ \\
\hline solid state relay & $\$ 6.45$ \\
\hline reset push button & $\$ 1.45$ \\
\hline power mount, power cord, and connection plug, etc. & $\$ 7.80$ \\
\hline PCB board manufacturing & $\$ 30.00$ \\
\hline miscellaneous & $\$ 3.21$ \\
\hline & $\$ 94.50$ \\
\hline
\end{tabular}


Table IV. Cost of the major components in the central server module.

\begin{tabular}{|c|c|}
\hline Component & Price \\
\hline XBee Explorer Dongle & $\$ 24.95$ \\
\hline XBee wireless transceiver & $\$ 19.00$ \\
\hline Total & $\$ 43.95$ \\
\hline
\end{tabular}

\section{Conclusions}

This paper describes a capstone senior design project that builds a wireless sensor and actuator network for monitoring the energy usage of $\mathrm{AC}$ appliances in a home environment. The design of the system prototype including two measurement nodes and a central server module is explained. The system prototype meets the design criteria. The implementation and performance analysis of this design project will be finished in the Spring semester of 2011 (The project is still ongoing when the paper is finalized). The design experience in this project will expose the students to up-to-date wireless and sensor technologies in an emerging real-world application.

\section{Bibliography}

1. Lawrence Berkeley National Laboratory, "Standby Power," http://standby.lbl.gov/standby.html, accessed March 2011.

2. SmartMeter ${ }^{\mathrm{TM}}$ - See Your Power, http://www.pge.com/myhome/customerservice/smartmeter, accessed March 2011.

3. TED - The Energy Detective, http://www.theenergydetective.com/, accessed in March 2011.

4. Google PowerMeter - Save energy. Save money. Make a difference, http://www.google.com/powermeter/, accessed in March 2011.

5. Kitt A Watt, http://www.p3international.com/products/special/P4400/P4400-CE.html, accessed in March 2011.

6. Panasonic, "Low Resistance Value Chip Resistors (Current Sensing Resistors)," http://industrial.panasonic.com/www-data/pdf/AOA0000/AOA0000CE7.pdf, accessed in March 2011.

7. Analog Devices, "Energy Metering IC with Integrated Oscillator and Reverse Polarity Indication," http:/www.analog.com/static/imported-files/data sheets/AD71056.pdf, accessed in March 2011.

8. IEEE 802.15.1 WPAN Task Group, http://www.ieee802.org/15/pub/TG1.html, accessed in March 2011.

9. IEEE 802.15.4 WPAN-LR Task Group, http://www.ieee802.org/15/pub/TG4.html, accessed in March 2011.

10. Digi International Inc. "XBee® \& XBee-PRO ${ }^{\circledR}$ ZB ZigBee® PRO RF Modules," http://www.digi.com/products/wireless/zigbee-mesh/xbee-zb-module.jsp, accessed in March 2011.

11. Sharp Microelectronics, "S116S02 Series/S216S02 Series, $\mathrm{I}_{\mathrm{T}}(\mathrm{rms}) \leq 16 \mathrm{~A}$, Zero Cross type, SIP 4pin, Triac output SSR,” http://media.digikey.com/pdf/Data\%20Sheets/Sharp\%20PDFs/S116,216S02.pdf, accessed in March 2011.

12. Bivar, "PLCC4 SMD Top View Package LED SMP4-RGY, RED/GREEN/YELLOW," http://www.bivar.com/images/cart/SMP4-RGY.pdf, accessed in March 2011.

13. Silicon Labs, "C8051F35x Analog-Intensive MCUs," http://www.silabs.com/products/mcu/mixedsignalmcu/Pages/C8051F35x.aspx, accessed in March 2011.

14. SparkFun, "XBee Explorer Dongle," http://www.sparkfun.com/products/9819, accessed in March 2011

15. C. Chen, C. Pomalaza-Ráez, and M. Oo, "Evaluation of IEEE 802.15.4 for Use in Smart Home Medical Care," in Proceedings of 2009 ASEE Annual Conference, June 2009, Austin, TX.

16. C. Chen and C. Pomalaza-Ráez, "Implementing and Evaluating a Wireless Body Sensor System for Automated Physiological Data Acquisition at Home," International Journal of Computer Science and Information Technology, vol. 2, no. 3, pp. 24-38, June 2010. 